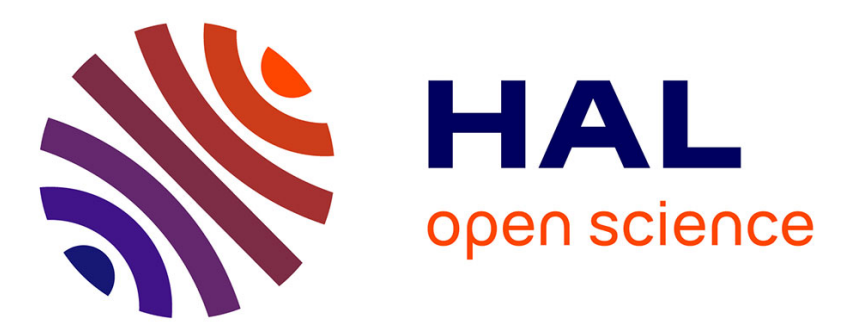

\title{
Polaronic interaction in a single modulation-doped GaAs quantum well with the
} Feynman-Hellwarth-Iddings-Platzman approximation G Martinez, J Wyzula, I Mohelsky, C Faugeras, M Orlita, M Potemski, A Riedel, R Hey, K J Friedland

\section{To cite this version:}

G Martinez, J Wyzula, I Mohelsky, C Faugeras, M Orlita, et al.. Polaronic interaction in a single modulation-doped GaAs quantum well with the Feynman-Hellwarth-Iddings-Platzman approximation. Physical Review B, 2021, 10.1103/PhysRevB.104.045312 . hal-03362670

\author{
HAL Id: hal-03362670 \\ https://hal.science/hal-03362670
}

Submitted on 8 Oct 2021

HAL is a multi-disciplinary open access archive for the deposit and dissemination of scientific research documents, whether they are published or not. The documents may come from teaching and research institutions in France or abroad, or from public or private research centers.
L'archive ouverte pluridisciplinaire $\mathbf{H A L}$, est destinée au dépôt et à la diffusion de documents scientifiques de niveau recherche, publiés ou non, émanant des établissements d'enseignement et de recherche français ou étrangers, des laboratoires publics ou privés. 


\title{
Polaronic Interaction in a single modulation doped GaAs quantum well revisited with the Feynman-Hellwarth-Iddings-Platzman approximation
}

\author{
G. Martinez, ${ }^{1}$ J. Wyzula, ${ }^{1}$ I. Mohelsky, ${ }^{1}$ C. Faugeras,${ }^{1}$ M. \\ Orlita, ${ }^{1,2}$ M. Potemski, ${ }^{1}$ A. Riedel, ${ }^{3}$ R. Hey, ${ }^{3}$ and K. J. Friedland ${ }^{3}$ \\ ${ }^{1}$ Laboratoire National des Champs Magnétiques Intenses, \\ CNRS-UGA-UPS-INSA-EMFL, 25, rue des Martyrs, 38042 Grenoble, France \\ ${ }^{2}$ Institute of Physics, Charles University in Prague, CZ-121 16 Prague, Czech Republic \\ ${ }^{3}$ Paul Drude Institute, Hausvogteiplatz 5-7, D-10117 Berlin, Germany
}

(Dated: July 12, 2021)

\begin{abstract}
Absolute far-infrared magnetotransmission experiments have been performed in magnetic fields up to $33.5 \mathrm{~T}$ on a series of single GaAs quantum wells doped with different electron concentrations. The transmission spectra have been simulated with a multilayer dielectric model. The imaginary part of the optical response function which reveals singular features related to the electron-phonon interactions has been extracted. In addition to the expected polaronic effects due to the longitudinaloptical phonon of GaAs additional interactions with interface phonons are observed. The main interaction is analyzed quantitatively with the Feynman-Hellwarth-Iddings-Platzman model which is shown to predict correctly the concentration of carriers beyond which the Fröhlich interaction is completely screened.
\end{abstract}

Pacs number: 78.67.De, 71.30.Fs,78.66.Fd

\section{INTRODUCTION}

Phonons, electrons and their interaction play a major role in the fundamental elementary excitations in solids. In general, that interaction occurs via the deformation potential experienced by the electrons moving across the vibrating nuclei. However, in a polar material, another fundamental interaction, the Fröhlich interaction, can exist between electrons and the longitudinal optical (LO) phonon giving rise to polaronic effects $(\mathrm{PE})$, the subject of many reports ${ }^{1-3}$. This process, driven by the free electron-dipole interaction, is quite strong in undoped material but very sensitive to the concentration of free carriers which screen the interaction. On a theoretical ground, PE were first studied by Lee and Pines ${ }^{4}$ and later by Feynman ${ }^{5}$. It was realized that this kind of interaction could not be properly handled by perturbation theory applied to the Hamiltonian of the problem, and required a global treatment. Such an approach was proposed by Feynman, Hellwarth, Iddings and Platzman ${ }^{6}$, and referred to, in the following, as the FHIP model. This model allows to solve analytically the path-integral method ${ }^{5}$ applied to the problem and, in turn, to evaluate ${ }^{7}$ quantitatively the contribution of the Fröhlich interaction to the imaginary part of the dielectric function. It is the goal of our paper to compare the predictions of the FHIP model with experimental results.

In quasi-two-dimensional GaAs (Q2D) based structures, there is evidence of polaronic effects ${ }^{8}$ but few experiments ${ }^{9,10}$ have focused on cyclotron resonance (CR) in the energy region above the reststrahlen band (RB) of GaAs (this requires for GaAs a magnetic field strength beyond $23 \mathrm{~T}$ ): these experimental conditions are essential to bring the CR transition energy in resonance with that of the LO phonon of the material, providing therefore a direct access to the imaginary part of the interaction. In the present case, a careful analysis of the optical transmission data obtained on a series of single GaAs quantum wells, imbedded between two GaAs/AlAs superlattices, as a function of magnetic fields up to $33.5 \mathrm{~T}$ allows a quantitative experimental evaluation of the imaginary part of the polaron interaction. These experimental results will be compared to the predictions of the FHIP model.

\section{CHARACTERIZATION OF SAMPLES AND EXPERIMENTAL PROCEDURE}

All samples discussed in this study have been grown by molecular beam epitaxy (MBE) on GaAs substrates. The active part of each sample is composed of a single doped GaAs quantum well $(\mathrm{QW})$ with a width $\mathrm{d}_{\mathrm{w}}$ of $13 \mathrm{~nm}$ sandwiched between two GaAs-AlAs short period superlattices with the Si-n-type doping performed symmetrically on both sides of the $\mathrm{QW}^{11}$. The $\delta$-doping is located in one of the GaAs layer of the superlattice at various distances from the $\mathrm{QW}$ allowing for varying the electron concentration $\mathrm{N}_{\mathrm{s}}$ in the QW. We have studied a series of samples with $\mathrm{N}_{\mathrm{s}}$ varying from $2.0 \times 10^{11} \mathrm{~cm}^{-2}$ to $9.0 \times 10^{11} \mathrm{~cm}^{-2}$ as measured, at low temperatures, by transport techniques. The mobility depends on the carrier concentration but always exceeds $10^{6} \mathrm{~cm}^{2} /(\mathrm{V} . \mathrm{s})$. For most of our samples, the optical transmission in the infra-red is completely obscured in the RB energy of the GaAs substrate, a region particularly important for our study. To circumvent this problem, one can grow, at the first step of the MBE process, a thick layer of AlAs which allows one, by chemical selective etching, to remove the epi-layer from the substrate. This epi-layer breaks into small flakes, floating at the surface of the chemical bath, which are transferred to a semi-insulating wedged Si substrate. This is called the lift-off process. The clear ad- 
vantage of the lift-off structures for infra-red studies has been previously demonstrated ${ }^{12}$. We have been able to investigate two lift-off samples with different carrier concentrations. For these samples, the carrier concentration was measured before the lift-off process. In general $\mathrm{N}_{\mathrm{s}}$ can also be evaluated by fitting at low fields the intensity of the CR transition: for non lift-off samples, the agreement between transport and optical data is very good within experimental errors. However, $\mathrm{N}_{\mathrm{s}}$ for lift-off samples, as deduced from optical data, is found significantly lower (about 10 percent) than the value obtained by transport data. Therefore the lift-off process relaxes strains in the epi-layer modifying the transfer of electrons between the dopants and the QW. The mobility, however, is not significantly affected because, within the experimental errors, the line-width of the cyclotron resonance at low fields is unchanged. The larger flakes have a typical "diameter" size of about $1.5 \mathrm{~mm}$ and have been used to perform absolute magneto-optical transmission measurements. We are going to compare these results with some other ones obtained on non lift-off samples and already partly published ${ }^{13}$. This will allow us to perform a complete consistent treatment of the data for all samples.

Far infra-red magneto-optical experiments have been performed at magnetic field strengths up to $33.5 \mathrm{~T}$ and at a fixed temperature of $1.8 \mathrm{~K}$. The sample insert was cooled down with a top black polyethylene window to keep the carrier concentration $\mathrm{N}_{\mathrm{s}}$ constant under illumination ${ }^{14}$. The light (provided and analyzed by a Fourier transform spectrometer) was delivered to the sample by means of light-pipe optics. All experiments were performed with non-polarized light, in the Faraday geometry with the wave vector of the incoming light parallel to the magnetic field direction and perpendicular to the plane of the samples. For lift-off samples, care has been taken to maintain the epilayer plane in the Faraday geometry. The spectral resolution was kept constant for most of the samples and equal to $2 \mathrm{~cm}^{-1}(\approx 0.25 \mathrm{meV})$. A Si bolometer was placed directly beneath the sample to detect the transmitted radiation. The response of this bolometer is strongly dependent on the magnetic field. Therefore, in order to measure the absolute transmission TA $(B, \omega)$, we used a rotating sample-holder and measured, for each value of $B$, a reference spectrum through either a hole for as-grown samples or a hole covered by a piece of Si substrate for lift-off samples (in both cases the diameter of the hole is adjusted to that of the sample). These spectra are, in turn, normalized with respect to $\mathrm{TA}(0, \omega)$ to obtain an absolute relative transmission spectrum $\operatorname{TR}(B, \omega)=\operatorname{TA}(B, \omega) / \mathrm{TA}(0, \omega)$ which only displays the magnetic field dependent features. With that presentation, any observed deviation of the intensity of the $\mathrm{CR}$ absorption with respect to that measured at low fields reveals, at the corresponding energy, an interaction which has to be explained.

\section{EXPERIMENTAL RESULTS FOR LOWER DOPED SAMPLES}

In Fig. 1, we display, for magnetic fields higher than $19 \mathrm{~T}$, the relative transmission spectra obtained for samples (a), (b) and (c) with a carrier concentration $\mathrm{N}_{\mathrm{s}}$ $=2.0 \times 10^{11} \mathrm{~cm}^{-2}, 3.0 \times 10^{11} \mathrm{~cm}^{-2}$ and $4.7 \times 10^{11} \mathrm{~cm}^{-2}$ respectively. Samples (a) and (b) are as-grown samples whereas sample (c) has been etched and transferred to $\mathrm{Si}$. We immediately note the additional wealth of information obtained with the lift-off sample (c).

The observed strong decrease of the CR line "intensity" for energies higher than $\hbar \omega_{L O}$ of GaAs is the very first characteristic of the Fröhlich interaction: this decrease corresponds to a broadening of the $\mathrm{CR}$ line with a width related directly to the imaginary part of the interaction. In addition, one also notices that the signal diminishes around $\hbar \omega_{\mathrm{TO}}$ of AlAs and also around $\hbar \omega_{\mathrm{TO}}$ of GaAs for sample (c). This tells us that, in order to simulate correctly the spectra, one needs to adopt a multi-layer dielectric model ${ }^{15}$ which includes the appropriate dielectric functions of all undoped barrier layers and the dielectric function $\bar{\varepsilon}$ of the doped QW. For the doped QW the CR active part $\varepsilon_{a}$, for instance, is written as:

$$
\varepsilon_{a}=\varepsilon_{L}-\frac{\omega_{p}^{2}}{\omega\left[\omega-\left(\omega_{N P}-\operatorname{Re}(\Sigma)\right)+\imath(\eta+\operatorname{Im}(\Sigma)]\right.}
$$

One has to include also the corresponding CR inactive part of the dielectric function to reproduce the data obtained with non-polarized light. In the above equation, $\varepsilon_{L}=\varepsilon_{\infty}-\frac{\left(\varepsilon_{0}-\varepsilon_{\infty}\right) \omega_{\mathrm{TO}}^{2}}{(\omega+\imath \gamma)^{2}-\omega_{\mathrm{TO}}^{2}}$ includes the high frequency dielectric function $\varepsilon_{\infty}$ and that of the GaAs lattice phonon, $\omega_{p}$ is the plasma frequency, $\omega_{N P}$ is the cyclotron frequency in the absence of polaron interaction but including (magnetic field dependent) non-parabolicity (NP) effects $^{16}$ and $\eta$ stands for a field independent contribution of the background defects to the width of the CR line. The effective mass $m^{*}$ entering the plasma frequency $\omega_{p}$ is calculated with the same NP model. $\Sigma(\omega)$ represents the self energy correction due to interactions between the electron gas and elementary excitations: they will be discussed further but we know from the response function theory that, whatever is the interaction described by $\Sigma(\omega), \operatorname{Re}(\Sigma(\omega))$ and $\operatorname{Im}(\Sigma(\omega))$ should be related by the Kramers-Kröning (KK) relations. The concept of self energy may be discussed because in general, in the absorption process, one should also include the electron-electron interaction: however, in the present case, all data which are analyzed are obtained for magnetic fields higher than $20 \mathrm{~T}$ where the filling factor $\nu<2\left(\nu=\mathrm{Ns}_{0} / B\right.$ and $\Phi_{0}$ is the flux quantum). In that case $^{17}$, the electronelectron interaction remains weak and negligible at least for lower doped samples.

Before fitting the transmission data, we note that the line-width and intensity of the CR transitions at low and high fields are practically the same. Hence we can assume that there is no loss of carrier density during the 

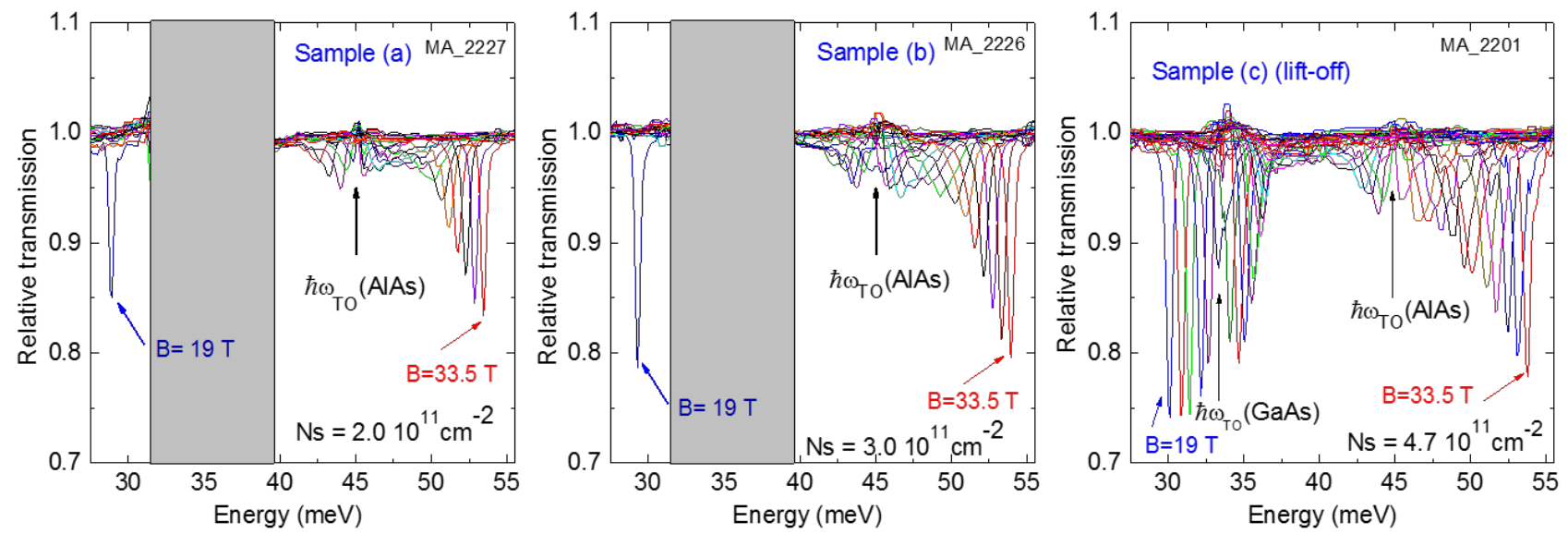

FIG. 1. (Color on line) Transmission spectra for samples (a), (b) and (c) as a function of the energy for different magnetic field values with steps of $0.5 \mathrm{~T}$. Spectra for samples (a) and (b) are displayed between $24 \mathrm{~T}$ and $33.5 \mathrm{~T}$ and spectra for sample (c) between $19 \mathrm{~T}$ and $33.5 \mathrm{~T}$.
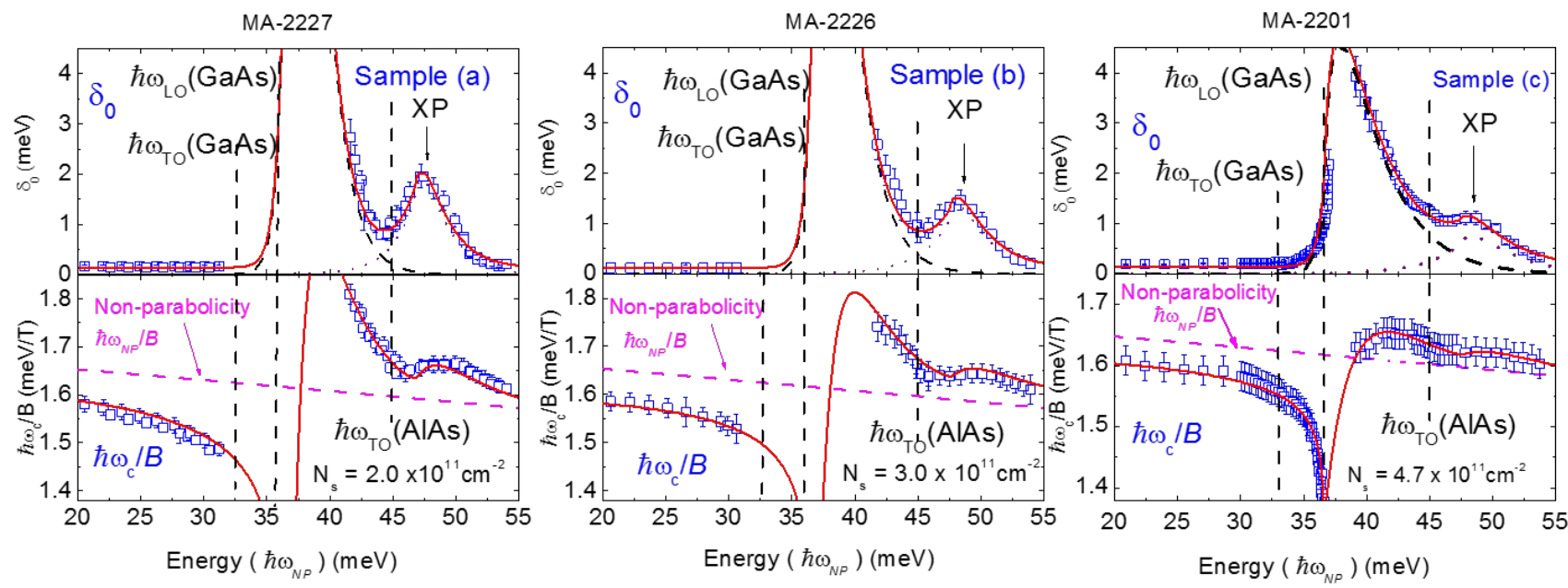

FIG. 2. (Color on line) Variation of the fitted parameters (open squares) $\delta_{0}$ (upper panels) and $\hbar \omega_{c} / B$ (lower panels) as a function of the energy $\left(\hbar \omega_{N P}\right)$. In the upper panels, the dotted and dashed lines are the simulated contributions of the different electron-phonon mechanisms to $\delta_{0}$. In the lower panels the dashed line is the contribution of the non-parabolicity effects to the variation of $\hbar \omega_{c} / B$. The continuous red lines simulate the variation of $\eta+\operatorname{Im}(\Sigma)$ in the upper panels and the corresponding KK transform in the lower panels.

magnetic field runs and that all interactions disappear at high fields. Therefore one can fit, for lower and very high fields, the contribution of NP effects and extract the linear dependance of $\omega_{N P}$ versus $B$ which will be used in the following analysis.

\section{FITTING THE TRANSMISSION DATA FOR LOWER DOPED SAMPLES}

In the fitting procedure, we first neglect the frequency dependence of $\Sigma$ and are left with two independent fitting parameters $\omega_{c}=\omega_{N P}-\operatorname{Re}(\Sigma)$ and $\delta_{0}=\eta+\operatorname{Im}(\Sigma)$ for each value of the magnetic field (and hence the cor- responding value of $\left.\hbar \omega_{N P}\right)$. The resultant parameters, $\hbar \omega_{c} / B$ and $\delta_{0}$, are displayed (open squares) in Figs. 2, upper and lower panels respectively, as a function of the energy $\hbar \omega_{N P}(B)$ (in these figures some typical error bars reflect the estimated uncertainties of the fitting procedure).

From these plots we notice that below $35 \mathrm{meV}, \delta_{0}$ is constant and small being mainly determined by $\eta$. Above this energy and approaching $\hbar \omega_{\mathrm{LO}}(\mathrm{GaAs})$, there is a sharp increase of $\delta_{0}$ observed in sample (c). There are two distinct contributions to $\delta_{0}$ : one lower field component starts very large but decreases rapidly at higher fields and a second component which starts from almost zero but goes through a maximum at a field $B$ corre- 


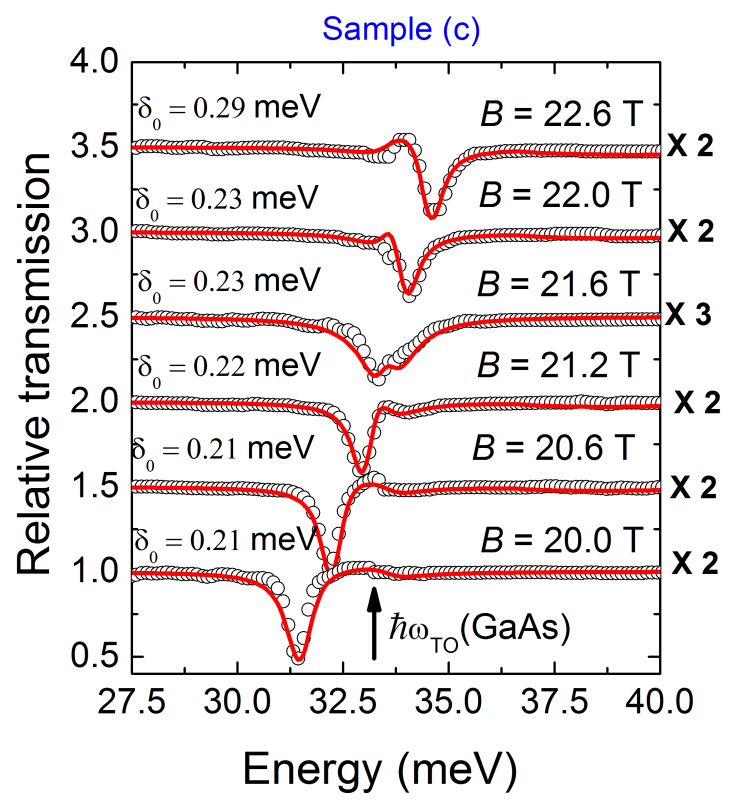

FIG. 3. (Color on line) Simulation of some experimental relative transmission spectra (open dots) with the calculated ones using the multi-layer dielectric model around the TO energy of GaAs (continuous red lines). Each curve is shifted by 0.5 with a scale enlarged as noted.

sponding to an energy of about $48 \mathrm{meV}$. This later contribution is seen in all samples : in accordance with a previous study ${ }^{13}$, we call it "XP". It will be further analyzed below.

Before discussing the main interaction, we notice that two singularities are also observed in Fig. 1 around the TO energies of GaAs and AlAs. When simulating the transmission spectra these singularities are perfectly reproduced, including the over-shoot, by the multi-layer dielectric model without invoking any singularity in $\delta_{0}$ at the corresponding energies. As an example, we show in Fig. 3, for sample (c), the simulation of the transmission data around $\hbar \omega_{\mathrm{TO}}(\mathrm{GaAs})$. The observed features around that energy are dielectric artefacts arising from the fact that $\omega_{\mathrm{TO}}$ is a pole of $\omega_{p h}$. Therefore, for lower doped samples, there is no interaction of the CR line with the TO phonon of GaAs.

We concentrate on the main contribution to the increase of $\delta_{0}$. We assign it to the polaronic interaction since it appears with a threshold energy corresponding to the GaAs LO phonon frequency (slab mode). This assignment is consistent with the observation that this interaction is very sensitive to the carrier concentration. To model this interaction we will used the FHIP model ${ }^{6}$ and the subsequent derivation of the conductivity with that model ${ }^{7}$. This derivation predicts, in principle, the emission of polarons at multiple values of $\hbar \omega_{\mathrm{LO}}$ : however, in the spirit of the Feynman polaron model, we restrict our analysis to the resonant emission of one po- laron. Then, for undoped polar material, $\operatorname{Im}\left(\Sigma^{\mathrm{Pol}}(\omega)\right)$ is written as $^{7}$ :

$$
\operatorname{Im}\left(\Sigma^{\mathrm{Pol}}(\omega)\right)=\frac{\mathrm{C} \times \omega_{\mathrm{LO}}^{2}}{\omega}\left|A_{0}(\omega)\right|^{1 / 2} e^{-\mathrm{R}\left|A_{0}(\omega)\right|} \Theta\left(A_{0}(\omega)\right)
$$

where $A_{0}(\omega)=\omega / \omega_{\mathrm{LO}}-1, \Theta(x)=1$ for $x>1$ or zero otherwise. Expressed in this way, the polaron contribution depends only on two parameters $\mathrm{C}$ and $\mathrm{R}$ both independent on energy. We have simulated this interaction with the curve, depicted in the upper panels of Fig. 2, with a dashed black line.

One has to simulate the contribution of the "XP" interaction which was observed in all GaAs based samples ${ }^{13}$. In fact, this interaction has also been observed in GaInAs $\mathrm{QW}^{18}$ and clearly assigned to the contribution of the symmetric interface modes developed in Q2D structures which are active in the infra-red ${ }^{19}$. The wave function of these symmetric interface modes does not vanish at the center of the QW (unlike the anti-symmetric modes) and therefore have a significant overlap with the wave function of the Q2D gas. However the interaction which is also of the Fröhlich type is governed by the Fröhlich constant of the barrier and therefore much less screened than the main polaronic interaction in the QW. Following the model proposed by Mori and Ando ${ }^{19}$, we simulate this interaction with a Lorentzian curve as depicted, in the upper panels of Fig. 2, with a dotted line. As a result, the energy of the maximum of the "XP" interaction, $E_{\max }^{X P}$, is found around $48 \mathrm{meV}$, an energy inside the $\mathrm{RB}$ energy of AlAs $\left(\hbar \omega_{\mathrm{TO}}(\mathrm{AlAs})=45 \mathrm{meV}\right.$ and $\hbar \omega_{\mathrm{LO}}$ $(\mathrm{AlAs})=50 \mathrm{meV})$ consistent with the assignment of the "XP" transition. In principle, one should expect, a similar contribution from the interface modes in the RB of GaAs but it is not observed (see Fig. 3) within the experimental errors in sample (c).

With all preceding approximations, one can fit the $\delta_{0}$ variation using, as a guide, the data points obtained from the first step of our analysis. This fitting provides the total self energy correction $\operatorname{Im}(\Sigma(\omega))=\operatorname{Im}\left(\Sigma^{P o l}(\omega)\right)+$ $\operatorname{Im}\left(\Sigma^{X P}(\omega)\right)$ which is displayed as thick red lines in the upper panels of Fig. 2.

Assuming these interactions satisfy the linear response function theory, $\operatorname{Im}(\Sigma(\omega))$ and $\operatorname{Re}(\Sigma(\omega))$ should be related by the Kramers-Kröning (KK) relations. Therefore, as a first constraint on the fitting process, the KK transform of $\delta_{0}(\omega)$ should reproduce the variation of $\hbar \omega_{c}(\omega) / B$ (open squares in the lower panels of Fig. 2). At that level, we compare also the experimental data with the variation of $\hbar \omega_{N P}(B) / B$ versus $\hbar \omega_{N P}(B)$ (magenta dashed lines) as obtained at the beginning.

Using this variation, one can refine the whole process: the fitted functions $\hbar \omega_{c} / B(\omega)$ and $\delta_{0}(\omega)$ are then inserted in the multi-layer dielectric model from which one can compute, for each value of $\mathrm{B}$, the TR spectrum and compare it to the corresponding experimental one. With that self-consistent treatment, we obtain an agreement, for all spectra and all samples, which is quite satisfactory (see 

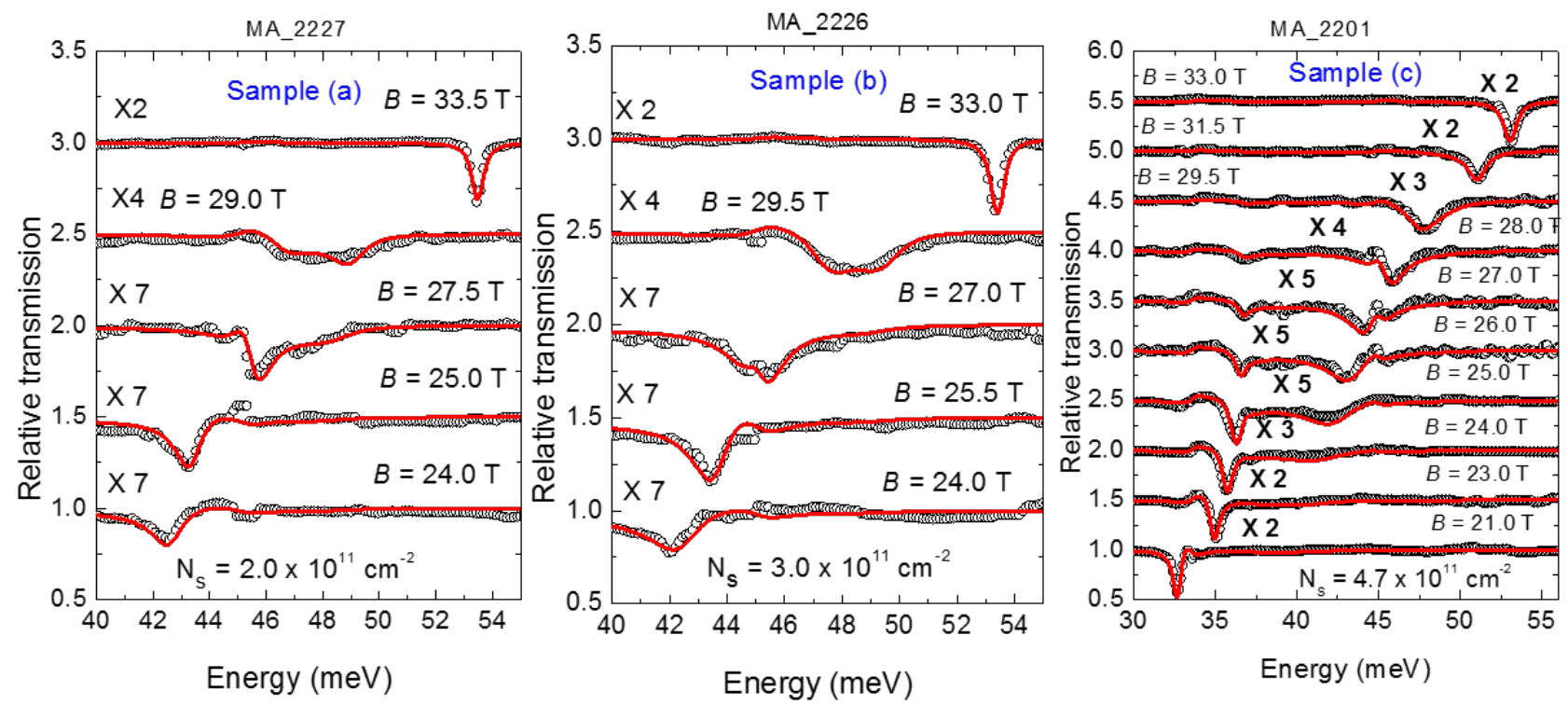

FIG. 4. (Color on line) Simulation, for samples (a),(b) and (c) of some experimental relative transmission spectra (open dots) with calculated ones (continuous red lines) using the multi-layer dielectric model where the fitted functions $\hbar \omega_{c}(\omega) / B$ and $\delta_{0}(\omega)$ have been inserted (see text). Each curve is shifted by 0.5 with a scale enlarged as specified.

Fig. 4) and lead us to think that the model has captured the main physical ingredients of the problem, including the quantitative aspects.

\section{DISCUSSION OF THE FITTING PARAMETERS FOR THE LOWER DOPED SAMPLES}

To treat the electron-phonon interaction in $3 \mathrm{D}$ compounds, Feynman ${ }^{5}$ introduced the path-integral method which applies the concept of "least-action" to solve the problem. This action describes the time evolution of the Lagrangian at all degrees of interaction. In practise, however, the problem can be solved analytically only if the interaction is harmonic.

The FHIP model uses the path-integral method and mimics the Fröhlich interaction (of Coulombic nature) by an harmonic interaction, with a force constant $v^{2}-w^{2}, v$ and $w$ being two parameters expressed in reduced units of $\omega_{\mathrm{LO}}$.

Expressed, as a function $v$ and $w$, the two fitting parameters $\mathrm{C}$ and $\mathrm{R}$ (Eq. 2) used in the preceeding section are not independent: $\mathrm{R}=\left(v^{2} / w^{2}-1\right) / v$ and $\mathrm{C}=\left(2 \alpha^{\mathrm{F}} / 3\right) \times(v / w)^{3}, \alpha^{\mathrm{F}}$ being the Fröhlich constant of the polar material (equal to 0.068 for GaAs). To take into account low dimensionality effects, we replace $\mathrm{C}$ by $\alpha_{\mathrm{Q} 2 \mathrm{D}} \times$ C. Following results from Hai et al. ${ }^{20}$, we have taken $\alpha_{\mathrm{Q} 2 \mathrm{D}}=1.2$. Within the philosophy of the FHIP model, the parameter $w$, specific of the phonon, should be the same for all samples and we have adjusted its value in order to reproduce the different $\mathrm{R}$ and $v$ values fitted
TABLE I. Parameters R and $v$ of the FHIP model for GaAs deduced from the fit of the data, assuming $w=0.175\left(\hbar \omega_{\mathrm{LO}}\right)$ and $\alpha^{\mathrm{F}}=0.068$

\begin{tabular}{ccccc}
\hline \hline Sample & $\alpha_{\mathrm{Q} 2 \mathrm{D}}$ & $\mathrm{N}_{\mathrm{s}}\left(10^{11} \mathrm{~cm}^{-2}\right)$ & $\mathrm{R}$ & $v\left(\hbar \omega_{\mathrm{LO}}\right)$ \\
\hline Sample a & 1.2 & $2.0 \pm 0.1$ & $25.7 \pm 0.5$ & $0.88 \pm 0.02$ \\
\hline Sample b & 1.2 & $3.0 \pm 0.1$ & $22.4 \pm 0.5$ & $0.76 \pm 0.02$ \\
\hline Sample c & 1.2 & $4.7 \pm 0.1$ & $12.7 \pm 0.5$ & $0.48 \pm 0.02$ \\
\hline \hline
\end{tabular}

experimentally and shown in table I for $w=0.175 \pm 0.005$.

In the FHIP model, as already said, the force constant of the harmonic interaction is proportional to $v^{2}-w^{2}$ entering the numerator of the parameter R. We have plotted, in Fig. 5, the variation of this quantity as a function of the carrier density $\mathrm{N}_{\mathrm{s}}$ of the QW. This variation clearly demonstrates the screening effects of the "polaronic" interaction. One can fit the data with a linear interpolation (dashed line in Fig. 5) justified by the fact that in Q2D systems the screening effects of the polaronic interaction varies linearly with $\mathrm{N}_{\mathrm{s}}{ }^{21}$. In this study performed for a QW width of $10 \mathrm{~nm}$, the screening is found to be complete for a critical value $\mathrm{Nsc1}$ of $\mathrm{N}_{\mathrm{s}}$ of the order of $10 \times 10^{11} \mathrm{~cm}^{-2}$ : this corresponds to a 3D concentration $\mathrm{N}_{3 \mathrm{D} 1}=10 \times 10^{17} \mathrm{~cm}^{-3}$. The present FHIP treatment provides a value $\mathrm{Nsc}=5.8 \pm 0.2 \times 10^{11} \mathrm{~cm}^{-2}$ corresponding to a $3 \mathrm{D}$ concentration $\mathrm{N}_{3 \mathrm{D}}=4 \times 10^{17} \mathrm{~cm}^{-3}$ significantly lower than $\mathrm{N}_{3 \mathrm{D} 1}$. This extra screening may be the manifestation of recoil effects naturally taken into account by the FHIP model. It turns out that, experimentally, the polaronic interaction around the LO phonon has practically disappeared for sample MA-1490 (sample (d)), see 


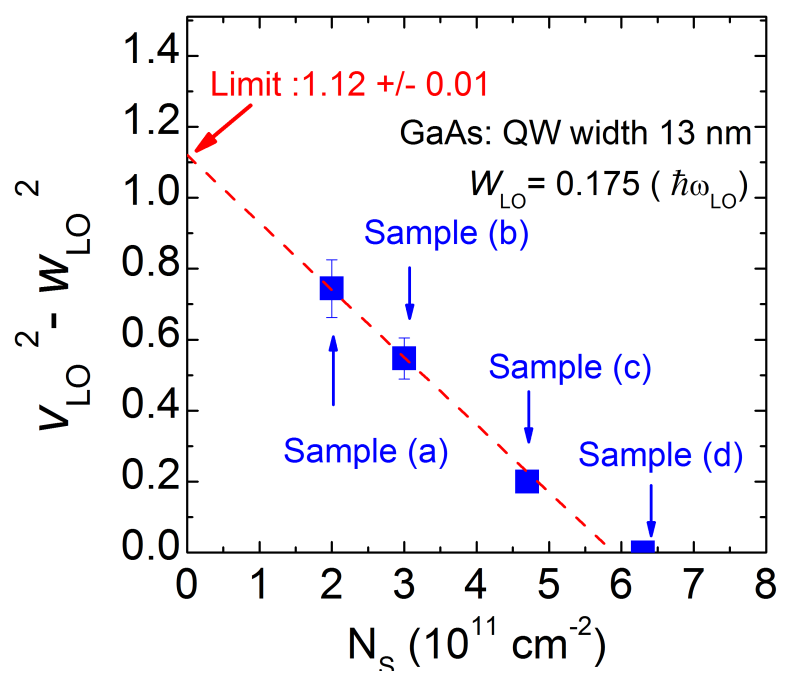

FIG. 5. (Color on line) Variation of $v^{2}-w^{2}$ as a function of the carrier density $\mathrm{N}_{\mathrm{s}}$ of the three samples (a), (b) and (c) (full squares). The dashed line is a linear interpolation of data.

next section) with $\mathrm{N}_{\mathrm{s}}=6.3 \times 10^{11} \mathrm{~cm}^{-2}$ consistent with our findings.

In the FHIP model, derived for undoped samples, the action $S_{F}$ of the Fröhlich interaction is mimicked by an harmonic action $S_{H}$ with a strength $v^{2}-w^{2}=\sqrt{8} \alpha^{F} / w$. Therefore from the limit at $\mathrm{N}_{\mathrm{s}}=0$, see Fig. 5 , one can derive the parameters describing the polaronic interaction, with the FHIP model, in GaAs for undoped samples : $\alpha^{F}=0.068, w=0.175 \pm 0.005$ and $v=1.065 \pm 0.05$.

Since the "XP" feature involves the Fröhlich interaction, one should also parameterized it with the FHIP model. However, within the experimental uncertainties, it is difficult to find the energy threshold beyond which it occurs without adding a significant number of broadening parameters. This may reflect the imperfections of the interfaces between the QW and the surrounding superlattices. Therefore, we fit this transition with a Lorentzian curve as proposed by Mori and $\mathrm{Ando}^{19}$. However this tells us that $E_{\max }^{X P}$ is an upper limit of the interface corresponding mode. This approach allows, nevertheless, to plot the intensity of the fitted Lorentzian versus $\mathrm{N}_{\mathrm{s}}$ and see that the "XP" contribution should disappear around the same critical value Nsc as that observed for the main polaronic interaction.

\section{EXPERIMENTAL RESULTS FOR HIGHER DOPED SAMPLES}

We report now, in Fig. 6 the experimental results obtained for sample (d) with $\mathrm{Ns}=6.3 \times 10^{11} \mathrm{~cm}^{-2}$. We see that already at $B=24.5 \mathrm{~T}$ the intensity of the $\mathrm{CR}$ line is recovered showing that the main polaronic interaction has disappeared. However beyond that field some

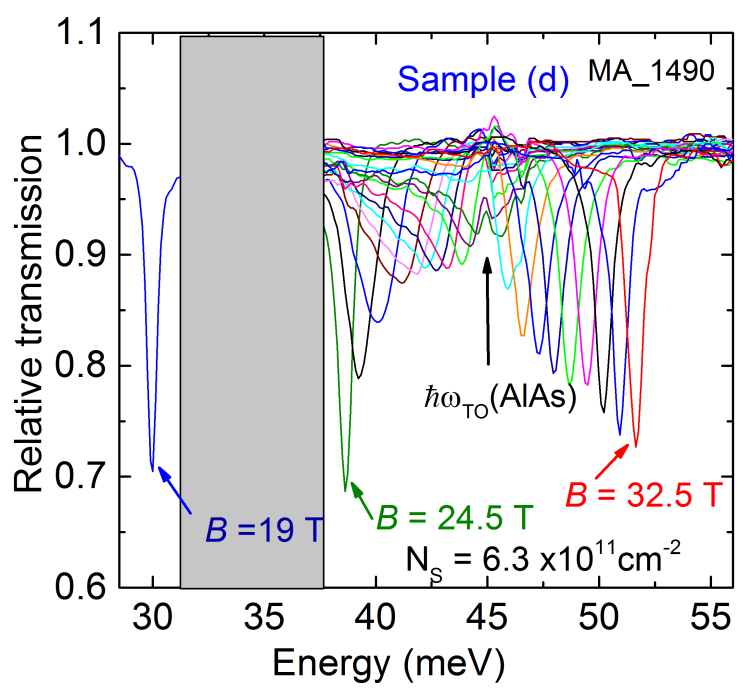

FIG. 6. (Color on line) Transmission spectra for sample (d) as a function of the energy for different magnetic field values.

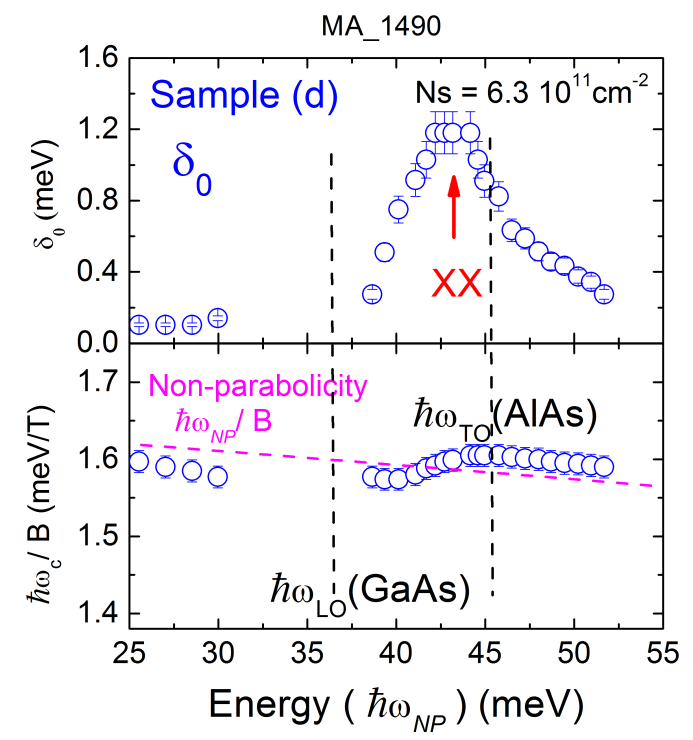

FIG. 7. (Color on line) Variation of the fitted parameters (open dots) $\delta_{0}$ (upper panel) and $\hbar \omega_{c} / B$ (lower panel), for sample $(\mathrm{d})$, as a function of the energy $\left(\hbar \omega_{N P}\right)$.

additional interaction remains. To characterize it, we have analyzed it using the standard model (Eq. 1) with $\Sigma(\omega)=0$. The resulting fitting parameters $\hbar \omega_{c} / B$ and $\delta_{0}$ are displayed in Fig 7 .

We note that $\delta_{0}$ is peaking at an energy "XX", a priori distinct from "XP", because it occurs at an energy below $\hbar \omega_{\mathrm{TO}}$ of AlAs that is outside the $\mathrm{RB}$ of this compound. We have performed experiments, not reported here, on other samples with $\mathrm{N}_{\mathrm{s}}=6.6 \times 10^{11} \mathrm{~cm}^{-2}$ and $7.1 \times 10^{11} \mathrm{~cm}^{-2}$ which displayed the same "XX" interac- 
tion below $\hbar \omega_{\text {TO }}$ of AlAs but with an intensity decreasing with $\mathrm{N}_{\mathrm{s}}$. We may question wether the doping of the QW can influence the solutions for the interface modes in such a structure.

The symmetric interface modes (only active in the infra-red range) of a GaAs QW sandwiched between two AlAs barriers are the solutions of the following equation $^{22}$ :

$$
\varepsilon_{\mathrm{GaAs}}(\omega) \tanh \left(\frac{Q \mathrm{~d}_{\mathrm{w}}}{2}\right)+\varepsilon_{\mathrm{AlAs}}(\omega)=0
$$

where $\mathrm{d}_{\mathrm{w}}$ is the thickness of the QW and $Q$ is the $2 \mathrm{D}$ wave vector of the interface phonon parallel to the interface. The solutions of Eq. 3 do not necessarily exist: they, in particular, depend on the values of the broadening parameters used to simulate the phonon part of the dielectric constant. We have used here, for these parameters, the same values as those adopted to simulate the transmission spectra. For undoped material, the solutions of this equation lie in the upper part of the RB of AlAs and the lower part of the RB of GaAs. We assume, in the following, that $Q \sim 1 / l_{B}, l_{B}$ being the magnetic field length. In the present case, $\varepsilon_{\mathrm{GaAs}}(\omega)$ is magnetic field dependent through the contribution of the cyclotron resonance which is as important as that of the phonon. We have calculated, for all samples, the solutions of Eq. 3 as a function of the magnetic field $B$ : results for sample (d) are displayed in Fig. 8. In this figure, we specify only those solutions (orange dots) which could lead to an extra diffusion of the CR transition: they need to be at an energy lower than the CR energy. Indeed the solutions are not very dependent on the carrier density: they always lie inside the RB of GaAs and that of AlAs and vary slightly with $B$ through the variation of the CR transition . The energies of the "XP" transition observed in the lower doped samples are in a good agreement with the solutions reported in Fig.8 inside the RB of AlAs. One may questions the validity of the present model because the barriers in our samples are in fact superlattices: we should therefore replace, in Eq.3, $\varepsilon_{\mathrm{AlAs}}(\omega)$ by an effective dielectric constant $\varepsilon_{\mathrm{eff}}(\omega)$ which can be calculated along the lines proposed by Merlin et al. ${ }^{23}$. It turns out that the results are very similar to those found with the simple model: in particular the solutions at high energies always lie inside the RB of AlAs. Therefore, with this approach, we do not have a physical explanation of the origin of the "XX" interaction displayed in Fig. 7. In addition, a closer look at the variation of $\hbar \omega_{c} / B$ as compared to that of $\hbar \omega_{N P} / B$ (lower panel of Fig. 7) leads us to think that certain additional effects, hidden in the $\mathrm{RB}$ of GaAs, should be present.

We then performed an additional set of experiments on a lift-off sample (MA 1200), referred in the following as sample (e), with a carrier density $\mathrm{Ns}=7.4 \times 10^{11} \mathrm{~cm}^{-2}$ . The corresponding relative transmission spectra are displayed in Fig. 9. (These spectra has been measured with a spectral resolution of $0.5 \mathrm{meV}$ ). We observed, as

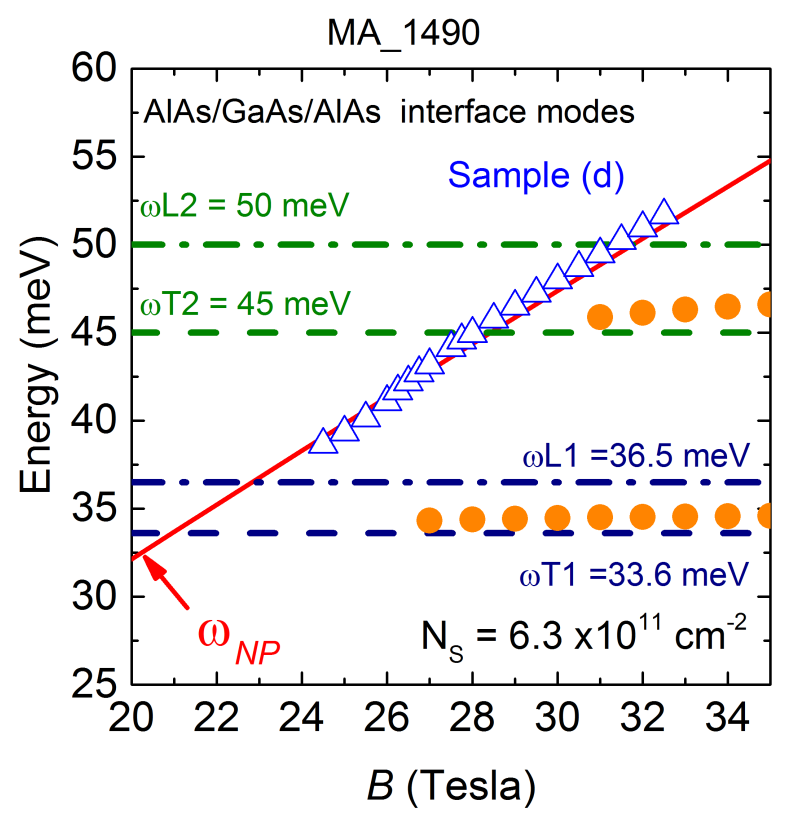

FIG. 8. (Color on line) Calculated energies, as a function of $B$, of the interface phonon modes for sample d (orange dots) expected to be active in the polaronic interaction. The red line is the contribution of the non-parabolicity effects $\omega_{N P}$ to the CR frequency as compared to the measured values $\hbar \omega_{c}$ (open blue triangles). $\omega \mathrm{T} 1$ and $\omega \mathrm{L} 1$ are the phonon frequencies of GaAs and $\omega \mathrm{T} 2$ and $\omega \mathrm{L} 2$ those of AlAs respectively.

for sample (c), a clear depletion of the CR "intensity" around $\hbar \omega_{\text {TO }}$ of GaAs and AlAs and a smaller one around the energy of the "XX" transition. However, here, the depletion in the RB of GaAs is, clearly, not only due to dielectric artefacts.

In Fig. 10 we plot the experimental results obtained for sample (e) (open black dots): they differ significantly from those displayed in Fig. 3 for sample (c). There is a clear interaction around $\hbar \omega_{\mathrm{TO}}$ of GaAs which almost disappears at $\hbar \omega_{\mathrm{LO}}$ of GaAs. This result is, in fact, not new and has already been reported ${ }^{12,24}$ in quantum wells with high electron density. Following these reports, Klimin and Devreese ${ }^{25}$ have developed a sophistical model showing that this effect could be due to the resonant magneto-polaron coupling of both types ( $\mathrm{GaAs}$ and AlAs) of interface phonon modes. Such a coupling is of electrostatic origin and does not imply the Fröhlich interaction. An interesting aspect is that this coupling, as a function of the carrier density, has a threshold below which it becomes relatively weak, explaining why it is not seen for low densities which could be the case for sample (c). In this study the authors focussed their attention on the splitting around $\hbar \omega_{\mathrm{TO}}$ of GaAs without discussing what could happen at higher energies. We think that such a mechanism could be, also, at the origin of the "XX" interaction. In that case, it is interesting to evaluate the relative strength of both interactions. 


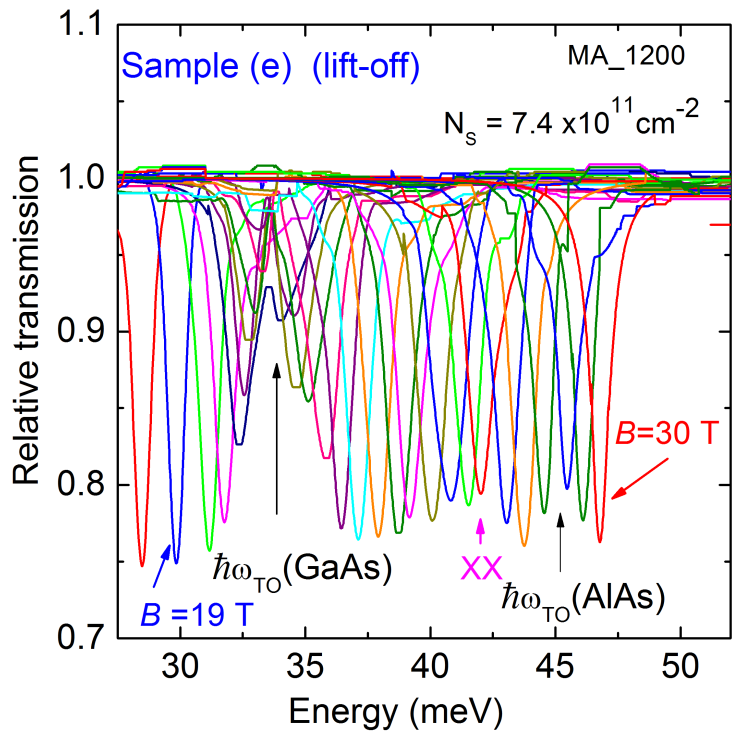

FIG. 9. (Color on line) Transmission spectra for sample (e) as a function of the energy for different magnetic field values.

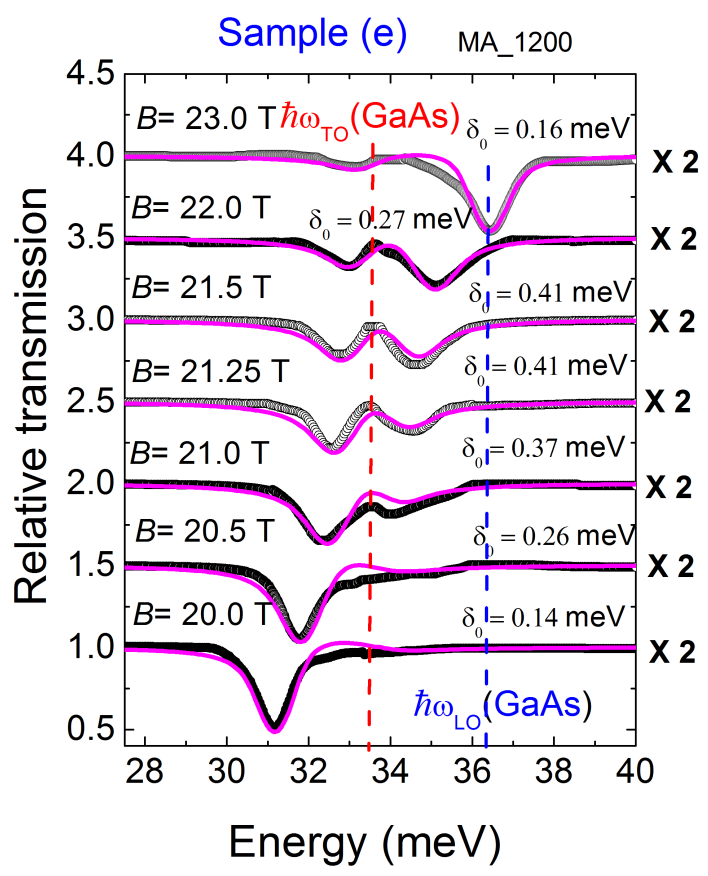

FIG. 10. (Color on line) Transmission spectra for sample (e) inside the RB of GaAs (open black dots). The magenta lines are the results of the fitting of the transmission curves with the model described in the text for an interaction parameter $V_{\mathrm{TO}}=1 \mathrm{meV}$.

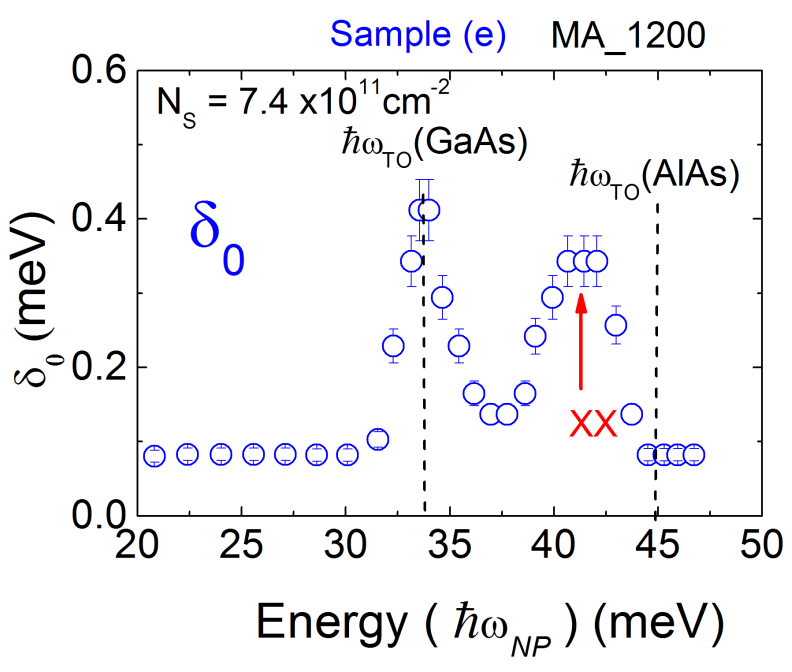

FIG. 11. (Color on line) Variation of the broadening parameters (open dots) $\delta_{0}$ for sample (e), as a function of the energy $\left(\hbar \omega_{N P}\right)$.

We have tried to quantify the interaction observed in Fig. 10 by simulating the transmission spectra with a simple model for the dielectric function which of course cannot be as simple as that given in Eq. 1. We show in Appendix how one can derive the active part of the dielectric function for a medium where the CR and TOphonon transitions interact with a parameter $V_{\text {TO }}$ to be fitted: the results are displayed in Fig. 10 as magenta lines with $V_{\mathrm{TO}}=1 \pm 0.1 \mathrm{meV}$. The fit is not perfect but gives an order of magnitude of the interaction which is believed reliable. The simulation of the experimental spectra with the modified model of the dielectric function, given in the appendix, allows to extract here also the imaginary part of the interaction. Results are displayed in Fig.11.

It is found that $\delta_{0}$ increases first around $\hbar \omega_{\mathrm{TO}}$ and then around the "XX" interaction similar to that found for sample (d). This treatment gives an idea of the relative strength of both interactions but is not self consistent: in particular, if we set $V_{\mathrm{TO}}=0$ we find a variation of $\delta_{0}$ around the "XX" interaction similar to that found here.

\section{CONCLUSION}

We have performed far infra-red magneto-optical transmission measurements on a series of n-type GaAs quantum wells, with a width $\mathrm{d}_{\mathrm{w}}$ of $13 \mathrm{~nm}$, doped at different electron concentrations $\mathrm{N}_{\mathrm{s}}$. Below a critical value Nsc of about $6 \times 10^{11} \mathrm{~cm}^{-2}$, the dominant features of the spectra are characteristic of the polaronic Fröhlich interaction with the LO phonon mode of GaAs and a similar one, called "XP", with the interface modes of the barrier. 
We have been able to extract from the data, the energy dependance of the real and imaginary parts of the selfenergy characteristic of both interactions. Results for the main interaction with $\hbar \omega_{\mathrm{LO}}$ of GaAs were quantitatively compared to the predictions of the FIHP model for different carrier concentrations. It was shown that, below Nsc, the model predicts correctly the carrier dependent screening of the Fröhlich interaction in such structures.

Beyond Nsc, new interactions appear first as a coupling of the CR transition with the TO phonon energy of GaAs and also, presumably, with a mixed mode, called "XX", of both interface phonons. These later results deserve a complete self consistent treatment to be understood.

\section{ACKNOWLEDGEMENTS}

The LNCMI-CNRS acknowledges the support of the EMFL.

\section{Appendix}

In order to include, in a self consistent way, the interaction of the cyclotron transition with that of the TO phonon, one has to express the active part of the dielectric function $\varepsilon_{a}$ (Eq.1) using the Green function formalism. In the absence of interaction the Green function
$G_{0}^{-1}$ is written as:

$$
G_{0}^{-1}=\left(\begin{array}{cc}
G_{e l}^{-1} & 0 \\
0 & G_{p h}^{-1}
\end{array}\right)
$$

where $G_{e l}^{-1}=\omega-\omega_{c}+\imath \delta_{0}$ and $G_{p h}^{-1}=\frac{(\omega+\imath \gamma)^{2}-\omega_{T O}^{2}}{\omega_{T O}}$ are the two propagators of the problem. Let us define, for the oscillator strengths of the optical transitions, a two dimensional vector $\vec{M}$ with components $M_{e l}=\sqrt{\omega_{p}^{2} / \omega}$ and $M_{p h}=\sqrt{\left(\varepsilon_{0}-\varepsilon_{\infty}\right) \omega_{T O}}$. With these definitions one can verify that $\varepsilon_{a}=\varepsilon_{\infty}-\overrightarrow{M^{T}} G_{0} \vec{M}$.

The simplest way to introduce the interaction between both optical transitions is to insert in the off-diagonal terms of Eq.A.1 a finite function that, for simplicity here, is taken as a constant $V_{T O}$. The perturbed Green function is written as:

$$
G^{-1}=\left(\begin{array}{cc}
G_{e l}^{-1} & V_{T O} \\
V_{T O}^{*} & G_{p h}^{-1}
\end{array}\right)
$$

from which we can derive the modified active part of the dielectric function $\varepsilon 1_{a}=\varepsilon_{\infty}-\overrightarrow{M^{T}} G \vec{M}$. This simple model has been used to fit the data reported in Fig.10
1 J. T. Devreese, J. Phys.: Condens. Matter 19, 255201 (2007) and references herein.

2 P. Gaal, W. Kuehn, K. Reimann, M. Woerner, T. Elsaesser and R. Rey, Nature 450, 1210 (2007).

$3 \mathrm{Yu}$. Chen, N. Regnault, R. Ferreira, Bang-Fen. Zhu, and G. Bastard, Phys. Rev. B 79, 235314 (2009).

4 T. D. Lee and D. Pines, Phys. Rev. 92, 883 (1953).

5 R. P. Feynman, Phys. Rev. 97, 660 (1955).

${ }^{6}$ R. P. Feynman, R. W. Hellwarth, C. K. Iddings and P. M. Platzman, Phys. Rev. 127, 1004 (1962).

7 F. M. Peeters and J. T. Devreese, Phys. Rev. B 28, 6051 (1983).

8 H. Sigg and P. Wyder, J. A. A. J. Perenboom, Phys. Rev. B 31, 5253 (1985)

9 Y. J. Wang, H. A. Nickel, B. D. McCombe, F. M. Peeters, J. M. Shi, G. Q. Hai, X. -G. Wu, T. J. Eustis and W. Schaff, Phys. Rev. Lett. 79, 3226 (1997).

10 C. Faugeras, G. Martinez, A. Riedel, R. Hey, K. J. Friedland and Yu. Bychkov, Phys. Rev. Lett. 92, 107403 (2004).

11 K. J. Friedland, R. Hey, H. Kostial, R. Klann, K. Ploog, Phys. Rev. Lett. 77, 4616 (1996)

12 A. J. L. Poulter, J. Zeman, D. K. Maude, M. Potemski, G. Martinez, A. Riedel, R. Hey and K. J. Friedland, Phys. Rev. Lett. 86, 336 (2001).

13 C. Faugeras, M. Orlita, S. Deutchlander, G. Martinez, P. Y. Yu, A. Riedel, R. Hey and K. J. Friedland, Phys. Rev. B 80, 073303 (2009).

14 When using a Fourier transform spectrometer the output light, with the appropriate beam splitter, contains a large part of visible light which is known to excite DX centers in GaAs based samples leading to increase, at low temperatures, the concentration of carriers. This spurious effect is eliminated by filtering the beam light with a black polyethylene window.

15 Yu. Bychkov, C. Faugeras and G. Martinez, Phys. Rev. B 70, 085306 (2004).

16 C. Hermann and C. Weisbuch, Phys. Rev. B 15, 823 (1977).

17 Y. A. Bychkov and G. Martinez, Phys. Rev. B 77, 125417 (2008).

18 M. Orlita, C. Faugeras, G. Martinez, S. A. Studenikin and P. J. Poole, Euro. Phys. Lett. 92, 37002 (2010).

19 N. Mori and T. Ando, Phys. Rev. B 40, 6175 (1989).

${ }^{20}$ G. Q. Hai, F. M. Peeters and J. T. Devreese, Phys. Rev. B 47, 10358 (1993).

21 WuXiaoguang, F. M. Peeters and J. T. Devreese, Phys. Rev. B 36, 9760 (1987).

22 M. Nakayama, M. Ishida, N. Sano , Phys. Rev. B 38, 6348 (1988).

23 R. Merlin, C. Colvard, M. V. Kein, H. Morkoc, A. Y. Cho, A. C. Gossard, Appl. Phys. Lett. 36, 43 (1980)

24 M. Ziesmann, D. Heitmann, L. L. Chang, Phys. Rev. B 35, 4541 (1987).

25 S. N. Klimin and J. T. Devreese, Phys. Rev. B 68, 245303 (2003). 